\title{
Extracellular vesicle-mediated transport of non-coding RNAs between stem cells and cancer cells: implications in tumor progression and therapeutic resistance
}

\author{
Muhammad Nawaz ${ }^{1,2}$ \\ ${ }^{1}$ Department of Rheumatology and Inflammation Research, Institute of Medicine, Sahlgrenska Academy, University of Gothenburg, Gothenburg, \\ Sweden; ${ }^{2}$ Department of Pathology and Forensic Medicine, Ribeirão Preto Medical School, University of São Paulo, Brazil \\ Correspondence to: Muhammad Nawaz. Department of Pathology and Forensic Medicine, Ribeirão Preto Medical School, University of São Paulo, \\ 14049900, Ribeirao Preto, Brazil. Email: nawazm.edu@gmail.com.
}

\begin{abstract}
Recent years have witnessed intensive progress in studying extracellular vesicles (EVs), both for understanding their basic biology and contribution to variety of diseases, biomarker discovery, and their potential as gene delivery vectors and source of innovative therapies. As such, stem cell-derived EVs have contributed significant knowledge which led to the development of cell-free therapies in regenerative medicine. Although, the role of stem cell-derived EVs in maintaining stemness, differentiation and repairing tissue injuries is relatively well-understood; however, knowledge about the contribution of stem cellderived EVs in cancer progression is just emerging. The aim of this review is, therefore, to discuss the recent developments in stem cell-derived EVs and tumor progression, placing a particular focus on noncoding RNA (ncRNA) mediated cancer progression and resistance against therapies. This includes the failure of normal hematopoiesis and the progression of myeloid neoplasms, enhanced capacity of cancer cells to proliferate and metastasize, and the conversion of normal cells into cancer cells, activation of angiogenic pathways and dormancy in cancer cells. These processes are shared by mesenchymal stem cells (MSCs), cancer stem like-cells and cancer cells in an intricate intratumoral network in order to create selfstrengthening tumor niche. In this context, EV-ncRNAs serve as mediators to relay bystander effects of secreting cancer stem cells (CSCs) into recipient cells for priming a tumor permissive environment and relaying therapeutic resistance. Collectively, this knowledge will improve our understandings and approaches in finding new therapeutic targets in the context of CSCs, which could be benefited through engineering EVs for innovative therapies.
\end{abstract}

Keywords: Cancer stem cells (CSCs); extracellular vesicles (EV); exosomes; extracellular RNA; non-coding RNA (ncRNA); microRNA; long non-coding RNA (lncRNA); tumor progression

Received: 31 August 2017; Accepted: 17 October 2017; Published: 24 October 2017.

doi: $10.21037 /$ sci.2017.10.04

View this article at: http://dx.doi.org/10.21037/sci.2017.10.04

\section{Introduction}

Stem cells are a class of undifferentiated pluripotent cells with substantial ability to self-renew and differentiate into variety of specialized cell types. Characteristically, stem cells are best known for their contribution in developmental processes and maintaining the regenerative programs for the body. However, defined populations of biologically discrete cells—so called cancer stem cells (CSCs) (1), could be responsible for initiating malignancy (that will be discussed in later sections). Understanding stem cell biology fully, is essential for gaining mechanistic insights into human diseases and their effects in regenerative process. The current applications of stem cells in translational medicine rely largely on their potency for repairing tissue damages and ameliorating organ functions. In this context, the best characterized are the mesenchymal stem cells 
(MSCs). According to the International Society for Cellular Therapy (ISCT), the MSCs are defined as plastic adherent cells with the capacity to differentiate into osteoblasts, chondrocytes, myocytes and adipocytes (2). The commonly considered sources of MSCs are bone marrow, adipose tissue, the umbilical cord, nervous tissue, dental pulp, amniotic fluid, the placenta, and menstrual blood $(3,4)$. MSCs derived from these sources represent remarkable differences in morphology, proliferation, self-renewal ability and differentiation potential (2). Importantly, their capacity to differentiate towards osteoblasts, chondrocytes, myocytes and adipocytes, their ability to be activated during injury and colonization to injury site offer a promising source in tissue regeneration. The benefits of stem cellbased therapies are evident from their success in improving the symptoms of many diseases; including, diabetes, osteoarthritis, spinal cord injury, myocardial injury, graft versus host disease, and bone repair shown in many clinical and preclinical models (5).

In addition to their characteristic functional attribution in regenerative process, the stem cells have also been implicated in cancer initiation and therefore, are designated as CSCs (1). CSCs possess substantial potential for clonal tumor initiation, long-term repopulation, and phenotypic plasticity preservation $(6,7)$. A number of cell surface markers, such as CD34, CD133, CD24, CD44, CD166, and epithelial cell adhesion molecule (EpCAM), are used to identify and enrich CSCs from several types of cancers (1). Although, the origin of CSCs and the prediction of their biological activity remained a debated issue $(1,8)$; and have been explained through CSC model (also known as a hierarchical model). This model posits that a defined population of biologically discrete cells is distinctively responsible for initiating malignancy (9), and that the tumor population is hierarchically arranged (10). Subsequently, the tumorinitiating activity can be enriched by sorting cells on the basis of intrinsic characteristics and may be prospectively isolated based on a specific cell surface marker (11).

Conversely, the stochastic model of cancer posits that all of the cells within a cancer have equal potential to act as cancer-initiating cells (CICs) with a potential to propagate the cancer [reviewed in (1)]. According to this model, tumor-initiating activity cannot be improved by sorting cells based on intrinsic characteristics and cannot be prospectively determined based on the local environment in which cells reside. This model assumes that the activities of CICs are governed by re-entry into the cell cycle, which is in fact a low-probability stochastic event, making it impractical to realistically identify the tumor-initiating subset.

Importantly, the secreted paracrine factors could play critical role in mediating crosstalk between the elements of the local stroma (microenvironment) and stem cells, whereby the cells may assertively orchestrate themselves in a given tumor niche and may exchange genetic material. The exchanges of biological material are vital to cancer development and could, therefore exhibit several features of CSCs (8). In this regard, the exchange of CSC-derived secretome with distinct CSC signatures presumably originated from different stem cell hierarchies could represent several features of their originating cells. Therefore, it is arguable that paracrine trophic factors originated from CSCs may reflect and recapitulate CSC features.

Recent body of evidence suggest that normal stem cells as well as the CSCs secrete small vesicles called extracellular vesicles (EVs), which serve as novel means of stem cellderived paracrine trophic factors (12). EVs are nano- and micro-sized vesicles that are ubiquitously produced from many perhaps all cell types under normal and pathological conditions. In fact, EVs are classified into heterogeneous populations of secreted vesicles (13-16). Among these, best characterized are the exosomes which are originated and produced via endocytic/exocytic pathway; and the microvesicles which bud off directly from the plasma membrane [for detailed mechanisms of biogenesis see (17)]. During their biogenesis, EVs are endogenously packaged with a repertoire of bioactive molecules such as proteins, transcriptional factors, lipids, carbohydrates, and nucleic acids including DNA, coding- and non-coding RNAs (ncRNAs) [reviewed elsewhere (18)]. Since these secreted vesicles transport bioactive molecules and disseminate biological signals, they act as mediators of intercellular communication between neighboring cells $(16,19,20)$, or bridging cellular channels between cells located apart (21). EVs could also transport their cargo particularly the circulating miRNAs to the cells of distant organs and may confer long distance inter-organ communication and metabolic regulation $(22,23)$.

Most commonly reported stem cells that secrete EVs include, embryonic stem cells (ESCs) and adult stem cells of different origins such as bone marrow mesenchymal stem cells (BMMSCs), adipose stem cells (ADSCs), human liver stem cells (HLSCs), induced pluripotent stem cells (iPSCs), and other tissue specific stem cells [reviewed in (12)]. It has been reviewed that EVs secreted from these stem cell sources potentially mimic the features of their secreting 
stem cell and are widely recognized as evolving factors in stem cell biology (12). The most frequently reported are the MSCs-derived EVs with potential therapeutic effects in tissue regeneration and organ healing (24-29).

Although, much has been documented about their participation in repair processes (29); less is known about how stem cell-derived EVs (from both normal stem cells and CSCs) contribute in cancer progression and mediate resistance to therapies. This review will, therefore, provide a detailed summary of recent developments of stem cell secreted EVs in tumor progression as well as tumor inhibition with mechanistic insights into stem cells, EVs, ncRNAs and intratumoral networking.

\section{RNA in stem cell derived EVs: implications in stem maintenance and differentiation}

Increasing body of evidence support that EVs mimic several features of their parent cells and have a profound effect on stem cell fate decisions (12). This includes the presence of bioactive molecules in EVs and their delivery between cells. However, the most profound paracrine regulatory effects of stem cell-derived EVs are elicited by transporting RNA species (30-34). First evidence was reported by Ratajczak and colleagues in 2006, which revealed that stem cellderived EVs contain mRNA transcripts for pluripotent transcriptional factors such as Nanog, Oct-4, HoxB4, and Rex-1, which can be horizontally transferred between cells, and favor the hematopoietic progenitor cells' expansion (35). Quesenberry and colleagues proposed that EV-mediated communication and exchange of genetic material is the continuum model of stem cell biology, where the differentiation decision of stem cells is conditioned by the cell cycle transit and the environmental stimuli (36). Stem cells help keeping population equilibrium between undifferentiated and the differentiated mature cells. This implies that the deficiency of differentiated mature cells in a particular tissue could be sensed by progenitors, which produce more progenies to be differentiated into mature cells. Such equilibrium could be facilitated by EV-mediated bidirectional exchange of genetic material, which favors stem cell populations to maintain a stable co-existence (12).

\section{Stem cell-derived ncRNA}

Stem cell-derived EVs not only transport coding RNA (i.e., mRNA), but also ncRNA species, which recapitulate the stem cell properties of their originating stem cells and serve as stem cells' extended paracrine messengers in maintaining stem cell features (37). It has been shown that MSCderived EVs are enriched in distinct ncRNA species such as miRNAs, tRNA and Piwi-interacting RNA (piRNAs), which contribute in maintaining stem cell potency (38), induction of cell survival and regulation of cell differentiation of cord blood hematopoietic stem cells (39). The comparison of transcriptomic (RNA-Seq) and proteomic profiles of ESCderived $\mathrm{EVs}$ and $\mathrm{EVs}$ from human BMMSC revealed distinctly different RNA profiles between EVs of two stem cell populations (40). The secretion of selective pattern of miRNAs from stem cells and their transfer to target cells via EVs raises enormous potential for stem cells to recapitulate lineage specific characteristics $(41,42)$.

Since ncRNAs are central to gene regulation and cellular fates, it can be speculated that most of the EV-mediated regulatory roles elicited in cells/organs are mediated through ncRNAs $(18,37)$. Indeed, ncRNAs are expressed in tissue-specific manner, precisely regulated and actively involved in variety of developmental processes (43-50), and the lineage specific commitments of stem cells and the maintenance of their characteristic features such as pluripotency, self-renewal, differentiation, and efficiency of cellular reprogramming are largely regulated by ncRNAs (51-60). Thus, the ncRNAs may govern the equilibrium between pluripotency and differentiation in stem cells, as well as lineage specific fate decisions $(61,62)$.

Additional roles for EV-miRNAs in cellular differentiation have been observed where EVmiR-486 delivery confers a rapid response to hypoxia in erythroleukemia cells by targeting Sirt1 gene, and modulates hypoxia-induced erythroid differentiation (63). Likewise, ESC-derived EVs could transport selective subset of miRNA and transcriptional factor related mRNAs which may induce pluripotency in their target cells and turn on early retinogenic program of differentiation (64). EVs could also contribute in hematopoietic progenitor cell mobilization through $\mathrm{EV}$-mediated transfer of miRNAs which downregulate vascular cell adhesion molecule (VCAM1) expression (65). Altogether, these studies support the idea that stem cells have evolved mechanisms for maintaining stem cell specific features at least, in part through EV-mediated dissemination of ncRNAs.

\section{NcRNA transport between stem cells and cancer cells: implications in tumor progression}

Several studies have demonstrated the role of stem cell- 
derived EVs in tumor progression [reviewed in (1,66-68)]; however, the role of stem cell-derived EVs carrying ncRNAs in cancer progression are only recently begging to be explored. Several layer of evidence has clarified that tumor initiation and progression through EV-mediated transport of abnormally expressed miRNAs could regulate oncogenic pathways (69-73). As such, EV-mediated dissemination of miRNAs may consequently contribute to the construction of premetastatic niche, metabolic reprograming, and the modulation of tumor microenvironment $(68,70,74-78)$.

It has been argued that $\mathrm{EV}$ s originated from normal stem cells possess regenerative properties, whereas those secreted from CSCs exhibit cancer-associated activities $(1,66,67,79)$. However, recent reports claim that EVs from normal stem cells could also have a profound effect on cancer progression and this is largely due to genetic content that is being disseminated and the conditions primed by stem cellderived EV-ncRNAs in recipient cells. As such, miRNAs and long non-coding RNAs (lncRNAs) disseminated from stem cells to cancer cells or from cancer cells to stem cells could induce bystander oncogenic affects in recipient cells and induce metastatic behaviors.

\section{Mechanisms}

\section{EV-miRNA dissemination and bystander effects}

It has been shown that MSCs express tumor supportive miRNA such as miR-21, and miR-34a that are secreted via EVs and delivered to cancer cells. The co-incubation of EVs with breast cancer cells in vitro allowed the enhanced angiogenesis and favored the cancer metastasis in recipient cells, whereas in vivo co-injections of EVs along breast cancer xenograft permitted the enhanced tumor size in a xenograft model (80). These effects were shown by EVmediated delivery of miRNAs. Glioma stem cells-derived EVs have also been shown to promote the angiogenic ability of endothelial cells through activation of miR-21/VEGF signaling pathway (81). Similarly, MSC-derived EVs could deliver miR-221 into human gastric cancer cells and modulate gene expression thereby allowing the proliferation and migration of recipient cancer cells (82). Interestingly, although EV-encapsulated miRNAs from prostate cancer bulk and CSCs reflect distinctly differential patterns; yet act cooperatively in cancer metastasis (83).

More lately, it has been reported that the miR-7977 in EVs is responsible for the hematopoietic dysfunctioning of MSCs by reducing the levels of poly(rc) binding protein
1 in myeloid neoplasms (84). This failure of normal hematopoiesis is subsequently linked with the progression of myeloid neoplasm. This is important to note that EVs are not only transferred from stem cells to cancerous cells but could also be delivered from cancer cells to stem cellsa reciprocal transfer. For instance, multiple myeloma (MM) cells were shown to deliver miR-146a into MSCs via EVs, which resulted into elevated level of cytokine secretion, which in turn created a conducive environment to facilitate cell viability and migration of MM cells (85). It has been shown that adult T-cell leukemia/lymphoma (ATL) cellderived EVs could deliver Tax, vascular endothelial growth factor (VEGF), and leukemia-related miRNAs such as miR-21 and miR-155 to MSCs, which alter their properties to create a more conducive milieu for leukemia (86). These both reports indicate the contribution of EVs in positive feedback loop between cancer cells and MSCs in order to favor tumor growth.

Recently, Kumar et al., has revealed that acute myeloid leukemia (AML) cells transform/remodel the bone marrow niche into a leukemia-permissive microenvironment through EV secretion and the downregulation of hematopoietic stem cell supporting factors (i.e., preparation of normal-hematopoiesis-suppressive microenvironment) (87). Engrafted AML-derived EVs or pre-conditioning with AML-derived EVs efficiently increased the MSC progenitors, blocked osteolineage development and bone formation in vivo and 'primed' the animals for accelerated AML growth. In addition, AMLderived EVs induced downregulation of hematopoietic stem cell supporting factors such as CXCL12, KITL, and IGF1 in BMMSC and reduced their ability to support normal hematopoiesis (87). Altogether, this study uncovers novel features of AML pathogenesis and unveils how AML cells create a self-strengthening leukemic niche that promotes leukemic cell proliferation and survival, while suppressing normal hematopoiesis through EV secretion.

\section{Networking between stem-like cells' subpopulations: beterogeneous intra-tumoral ecosystem}

Recent body of evidence suggests that EVs and miRNAs convey biological material that could rearrange the molecular landscape within the heterogeneous intratumoral ecosystem in a cell type-specific manner as shown by characterization of miRNA secretion in phenotypically diverse subpopulations of glioblastoma stem-like cells (GSCs). It was shown that the intratumoral exchange of 
miRNA could augment the heterogeneity of patient-derived GSCs. This was reflected in highly heterogeneous profile of miRNA expression in GBM subtypes (88). Moreover, it was reported that the expression of the subtype-enriched miRNAs such as miR-128 within transcriptionally and phenotypically diverse subpopulations of patientderived GSCs is a potent mechanism of bidirectional transitions between GBM subpopulations (89). This could consequently result in intermediate hybrid stages, highlighting highly intricate intra-tumoral networking. In addition to persistence of intra-tumoral heterogeneity, the loss of subtype characteristics in the tumor initiating cell compartment may contribute potent role in therapeutic resistance. This was shown in a recent study by Rak and colleagues where individual tumors derived from the same isogenic GSC line were able to express divergent and complex profiles of temozolomide resistance markers (90). Authors concluded that intrinsic changes in the tumor initiating cell compartment may include loss of subtype characteristics and reciprocal alterations in sensitivity to chemo- and radiation therapy. This divergent evolution of chemo-resistance is reflected in EVs (90).

Recently, Figueroa et al., has reported that glioma associated-human MSCs (GA-hMSCs)-derived EVs facilitate tumor supporting activities of GSCs by transferring glioblastoma specific miR-1587 between cells (91). Additionally, the comparison of miRNA content of EVs isolated from breast patients revealed stem cell-like properties and metastatic signature from patients with worse prognosis (92). This data supports the view that cancer cells with stem-like properties and their EVs could potentially aggravate tumor progression and metastasis.

\section{Resistance to chemotherapies and tumor progression}

Emerging studies indicated that CSCs represent a subpopulation of cells within the tumor that is responsible for chemotherapeutic resistance. In this context, one of the emerging roles of stem cell-derived EVs is their contribution to mediate cancer cell resistance against therapies (18), and promote tumor growth. The most propelling examples are those observed from MSCs. It has been recently demonstrated that breast cancer cells educate MSCs to release EVs with distinct miRNA content, such as miR-222/223, which promotes quiescence in a subset of cancer cells and confers drug resistance (93). In an immunodeficient mouse model of dormant breast cancer, the systemic administration of MSC-derived EVs loaded with antagomiR-222/223 efficiently sensitized breast cancer cells to carboplatin-based therapy and increased host survival. This indicates the role of EV-miRNA to mediate regulatory interactions between MSCs and breast cancer cells in the evolution of tumor dormancy and resurgence in the local metastatic microenvironment (93). Interestingly, this approach may favor the development of nontoxic therapeutic strategy to target dormant breast cancer cells for enhancing drug sensitivity and tumor monitoring.

Cancer cell derived EV-miRNAs have been implicated in the maintenance of stem cell property and chemotherapeutic resistance in cancer cells. For instance upregulation of miR-196b-5p in colorectal cancer (CRC) tissues enhances the spheroids formation ability, the fraction of stem-like subpopulations and the expression of stem cell factors (94). Moreover, miR-196b-5p is highly enriched in the serum EVs of patients whereby elevated expression of miR-196b-5p promotes stemness and chemoresistance of CRC cells against 5-fluorouracil via targeting SOCS1 and SOCS3 of STAT3 signaling pathway, and subsequent activation of STAT3 signaling pathway.

MSCs-derived EVs contain over expressed miRNA-23b, which promotes dormancy in metastatic breast cancer cells by suppressing MARCKS target gene (95). EVs transfer miRNAs to breast cancer cells which could potentially regulate chemoresistance in recipient cancer cells as well as in CSCs (96). In another example, the miRNAs from cancer-associated adipocytes and cancer associated fibroblasts could be transported to ovarian cancer cells via EVs, which confer paclitaxel resistance by suppressing apoptotic peptidase activating factor 1 (APAF1) in recipient cancer cells (97). In addition to their contribution as mediators of chemoresistance, EVs may also facilitate chemosensitivity in cancer cells. EVs derived from ADMSCs are implicated in promoting chemosensitivity in HCC and favor antitumor efficacy of chemotherapeutic agents in recipient cancer cells (98). The AD-MSCs transfected with miR-122, tether miRNA into EVs, that is taken by HCC upon co-incubation of EVs with HCC cells and promotes increased sensitivity to chemotherapeutic agents (98). Collectively these studies could offer a potential rational for combining $\mathrm{EV}$-mediated anti-miRNA strategies with conventional chemotherapy against cancer.

\section{LncRNAs in stem cell-derived EVs and tumor progression}

Although, the role of EV-associated lncRNAs from various cell types has been demonstrated in the progression of 
cancer and resistance to therapies (18); however, there is limited knowledge about the contribution of stem cellderived EV-lncRNAs in cancer progression. Recent studies demonstrate that lncRNAs in EVs are transferred in both directions i.e. from stem cells to cancer cells as well as from cancer cells to stem cells and act differently in cancer progression. For instance, CD $90^{+}$liver cancer cells modulate endothelial cell phenotype and promote angiogenesis through EV-contained H19 lncRNA (99). However, EVs from parental hepatoma cells lacking CD90 do not exhibit such features, indicating that such features of EV-linked $\mathrm{H} 19$ are exclusive to $\mathrm{CD} 90^{+}$cancer stem-celllike cells. Conversely, cancer cells may transfer lncRNA molecules to stem cells and modulate their behaviors. For example, MSCs treated with lung cancer-derived EVs revealed that EVs from cancer cells could initiate global changes in lncRNA expression in MSCs (100).

\section{Anti-tumor effects of stem cell-derived EV-miRNAs and tumor inbibition}

Interestingly, in addition to their participation in cancer progression, the stem cell-derived EV-miRNAs are well appreciated for their contribution in tumor inhibition. For instance, EVs from a conditioned medium of ADMSCs could induce apoptosis signaling in cancer cells by upregulating different pro-apoptotic signaling molecules, such as BAX, CASP9, and CASP3, and downregulating the anti-apoptotic protein BCL2 (101). EVs treatment exhibited the reduced viability of ovarian cancer cells. Additionally, sequencing of EV-RNAs revealed a rich population of miRNAs, which exhibit anti-cancer activities by targeting different molecules associated with cancer survival (102). These findings are in agreement with previously reported tumor inhibitory activities of ADMSC derived EVs (101). In fact, miR-145 from ADMSC derived EVs could extend the inhibitory effects of MSCs on prostate cancer by blocking the activities of anti-apoptotic Bclxl protein, followed by increased cell apoptosis of prostate cancer cells (101).

EVs-mediated delivery of selective miRNAs from HLSCs may reprogram HepG2 hepatoma and primary hepatocellular carcinoma cells by inhibiting their growth and survival in vitro (103). In vivo intra-tumor administration and uptake of HLSC-EVs in severe combined immune deficiency (SCID) mice may induce regression of ectopic tumors developed through the delivery of miRNAs to tumor cells. The antitumor effect of HLSC-EVs and
miRNA delivery was also observed in tumors other than hepatoma such as lymphoblastoma and glioblastoma, which showed that the delivery of selected miRNAs by stem cellEVs efficiently inhibits tumor growth and may stimulate apoptosis (103).

Fareh et al. has shown that primary glioma cells transport miR-302-367 to neighboring GBM cells via EVs, which could modulate the expression of stemness markers, as well as the proliferation and the tumorigenicity of recipient GBM cells (104). Importantly, EV-mediated transfer of miR-302-367 elicited the inhibition of its targets such as CXCR4/SDF1, SHH, cyclin D, cyclin A and E2F1 in recipient GBM cells indicating their effect on cell cycle inhibition. Furthermore, the orthotopic xenograft of miR302-367-expressing cells together with GSCs efficiently altered the tumor development in mice brain (104). This indicates that EVs could be exploited as vectors of cell cycle inhibition in cancer cells. MSCs transfected with miR-146b produce EVs loaded with exogenous miR-146b; whereas, intra-tumor injection of miR-146 containing EVs could significantly reduce glioma xenograft growth in a rat model of primary brain tumor (105). MSC-derived EVs transfer miR-100 to breast cancer cells which down-regulates VEGF expression (106). Moreover, downregulation of VEGF expression by MSC-derived EV-miR-100 could affect the vascular behavior of endothelial cells in vitro. This indicates that EV-mediated transfer of miR-100 to cancer cells paracrine effects of MSC-derived EVs in modulating vascular responses (angiogenesis) within the microenvironment of cancer cells, thus offering a vector of targeting cancer angiogenesis.

EVs derived from menstrual stem cells (MenSCs) are capable to inhibit prostate tumor through inhibiting reactive oxygen species (ROS) (107). In fact, the endometrial cells are fine regulators of the angiogenic process during the menstrual cycle that includes an angiostatic condition. EVs from MenSCs could induce a reduction in VEGF secretion and NF- $\kappa \mathrm{B}$ activity in prostate cancer cells. The ROS production was reduced in $\mathrm{EV}$-treated cells, suggesting that the inhibition of the intracellular ROS influences both VEGF and NF- $\kappa \mathrm{B}$ pathways (including the inhibition of VEGF and HIF- $1 \alpha$ expression). This indicates that the MenSCs-EVs suppress the secretion of pro-angiogenic factors by prostate cancer cells in a ROS-dependent manner. Importantly, the anti-angiogenic effect of EVs is specific to the menstrual cell source, as the BMMSCderived EVs showed an opposite effect on the VEGF and bFGF expression in tumor cells. Since the MenSCs-derived 
EVs act as blockers of the tumor-induced angiogenesis, therefore they could be suitable candidates for anti-cancer therapies. In a previous study, MSC-derived EVs were shown to significantly down-regulate the VEGF expression in tumor cells, which lead to inhibition of angiogenesis in vitro and in vivo (108). It was shown that MSC-derived $\mathrm{EVs}$ are enriched in anti-angiogenic miRNA-16 that is internalized by breast cancer cells and suppress VEGF expression thereby favoring the inhibition of angiogenesis in recipient tumor cells.

\section{Concluding remarks}

EVs secreted from MSCs, cancer-like stem cells, and cancer cells actively disseminate biologically active cargos, which could facilitate tumor progression directly or through relaying bystander effects. The tumor promoting activities are shared between normal stem cells of various tissue origins, cancer stem like-cells (i.e., CSCs), and cancer cells in an intricate intratumoral network to create selfstrengthening tumor niche, where EV-ncRNAs serve as mediators to transmit bystander effects of secreting cells into recipient cells for priming a conducive environment. It could be speculated that EV-based cancer-initiating activities of CSCs (i.e., identification of cancer initiating cells-CICs), and functional enrichment could add a layer of valuable knowledge to explain cancer-initiating models (1). In this context, CSC-subpopulation specific EV-ncRNAs could contribute new knowledge for resolving the existing controversies in cancer-initiating activities and enrichment, which are hotly debated issues in CSC model and the stochastic cancer model.

Given that the MSC-derived or cancer cell-derived EV-ncRNAs facilitate positive feedback loop between cancer cells and MSCs to create a tumor permissive microenvironment; this knowledge could be a potential target to create a suppressive microenvironment for targeting tumor growth. Indeed, EVs are efficient biological vehicles of cargo delivery between cells. This indicates that EVs, particularly MSC-derived EVs could be engineered for the delivery of anti-cancer drugs and genes (antagonistic miRNAs or siRNA) for CSC targeting therapy or MSC-based targeting therapies for monitoring cancer pathways (66). However, the procedures of obtaining EVs from desired sources for therapeutic purposes need robust and sensitive capture techniques and the endorsement of therapeutic potencies of EVs in clinical settings needs rigorous validation in model animals.

\section{Acknowledgements}

M Nawaz acknowledges Sao Paulo Research Foundation (FAPESP), Proc. No. 12/24574-3), and Coordination for the Improvement of Higher Education Personnel (CAPES, Proc. No. 99999.007057/2015-06), Brazil.

\section{Footnote}

Conflicts of Interest: The author has no conflicts of interest to declare.

\section{References}

1. Fatima F, Nawaz M. Stem cell-derived exosomes: roles in stromal remodeling, tumor progression, and cancer immunotherapy. Chin J Cancer 2015;34:541-53.

2. Dominici M, Le Blanc K, Mueller I, et al. Minimal criteria for defining multipotent mesenchymal stromal cells. The International Society for Cellular Therapy position statement. Cytotherapy 2006;8:315-7.

3. Eirin A, Riester SM, Zhu XY, et al. MicroRNA and mRNA cargo of extracellular vesicles from porcine adipose tissue-derived mesenchymal stem cells. Gene 2014;551:55-64.

4. Hass R, Kasper C, Bohm S, et al. Different populations and sources of human mesenchymal stem cells (MSC): A comparison of adult and neonatal tissue-derived MSC. Cell Commun Signal 2011;9:12.

5. Wei X, Yang X, Han ZP, et al. Mesenchymal stem cells: a new trend for cell therapy. Acta Pharmacol Sin 2013;34:747-54.

6. Matchett KB, Lappin TR. Concise reviews: cancer stem cells: from concept to cure. Stem Cells 2014;32:2563-70.

7. Beck B, Blanpain C. Unravelling cancer stem cell potential. Nat Rev Cancer 2013;13:727-38.

8. Bjerkvig R, Tysnes BB, Aboody KS, et al. Opinion: the origin of the cancer stem cell: current controversies and new insights. Nat Rev Cancer 2005;5:899-904.

9. Kreso A, Dick JE. Evolution of the cancer stem cell model. Cell Stem Cell 2014;14:275-91.

10. Bonnet D, Dick JE. Human acute myeloid leukemia is organized as a hierarchy that originates from a primitive hematopoietic cell. Nat Med 1997;3:730-7.

11. Reya T, Morrison SJ, Clarke MF, et al. Stem cells, cancer, and cancer stem cells. Nature 2001;414:105-11.

12. Nawaz M, Fatima F, Vallabhaneni KC, et al. Extracellular Vesicles: Evolving Factors in Stem Cell Biology. Stem 
Cells Int 2016;2016:1073140.

13. Crescitelli R, Lasser C, Szabo TG, et al. Distinct RNA profiles in subpopulations of extracellular vesicles: apoptotic bodies, microvesicles and exosomes. J Extracell Vesicles 2013;2:20677.

14. van der Pol E, Boing AN, Gool EL, et al. Recent developments in the nomenclature, presence, isolation, detection and clinical impact of extracellular vesicles. J Thromb Haemost 2016;14:48-56.

15. Lai RC, Tan SS, Yeo RW, et al. MSC secretes at least 3 EV types each with a unique permutation of membrane lipid, protein and RNA. J Extracell Vesicles 2016;5:29828.

16. Mateescu B, Kowal EJ, van Balkom BW, et al. Obstacles and opportunities in the functional analysis of extracellular vesicle RNA - an ISEV position paper. J Extracell Vesicles 2017;6:1286095.

17. Nawaz M, Camussi G, Valadi H, et al. The emerging role of extracellular vesicles as biomarkers for urogenital cancers. Nat Rev Urol 2014;11:688-701.

18. Fatima F, Nawaz M. Vesiculated long non-coding RNAs: Offshore packages deciphering trans-regulation between cells, cancer progression and resistance to therapies. NonCoding RNA 2017;3:10.

19. Valadi H, Ekstrom K, Bossios A, et al. Exosome-mediated transfer of mRNAs and microRNAs is a novel mechanism of genetic exchange between cells. Nat Cell Biol 2007;9:654-9.

20. Raposo G, Stoorvogel W. Extracellular vesicles: exosomes, microvesicles, and friends. J Cell Biol 2013;200:373-83.

21. Nawaz M, Fatima F. Extracellular Vesicles, Tunneling Nanotubes, and Cellular Interplay: Synergies and Missing Links. Front Mol Biosci 2017;4:50.

22. Thomou T, Mori MA, Dreyfuss JM, et al. Adipose-derived circulating miRNAs regulate gene expression in other tissues. Nature 2017;542:450-5.

23. Fatima F, Nawaz M. Long Distance Metabolic Regulation through Adipose-Derived Circulating Exosomal miRNAs: A Trail for RNA-Based Therapies? Front Physiol 2017;8:545.

24. Arslan F, Lai RC, Smeets MB, et al. Mesenchymal stem cell-derived exosomes increase ATP levels, decrease oxidative stress and activate PI3K/Akt pathway to enhance myocardial viability and prevent adverse remodeling after myocardial ischemia/reperfusion injury. Stem Cell Res 2013;10:301-12.

25. Tan CY, Lai RC, Wong W, et al. Mesenchymal stem cell-derived exosomes promote hepatic regeneration in drug-induced liver injury models. Stem Cell Res Ther
2014;5:76.

26. Lai RC, Chen TS, Lim SK. Mesenchymal stem cell exosome: a novel stem cell-based therapy for cardiovascular disease. Regen Med 2011;6:481-92.

27. Kordelas L, Rebmann V, Ludwig AK, et al. MSC-derived exosomes: a novel tool to treat therapy-refractory graftversus-host disease. Leukemia 2014;28:970-3.

28. Emanueli C, Shearn AI, Angelini GD, et al. Exosomes and exosomal miRNAs in cardiovascular protection and repair. Vascul Pharmacol 2015;71:24-30.

29. Fatima F, Nawaz M. Nexus between extracellular vesicles, immunomodulation and tissue remodeling: for good or for bad? Ann Transl Med 2017;5:139.

30. Baj-Krzyworzeka M, Szatanek R, Weglarczyk K, et al. Tumour-derived microvesicles carry several surface determinants and mRNA of tumour cells and transfer some of these determinants to monocytes. Cancer Immunol Immunother 2006;55:808-18.

31. Aliotta JM, Pereira M, Johnson KW, et al. Microvesicle entry into marrow cells mediates tissue-specific changes in mRNA by direct delivery of mRNA and induction of transcription. Exp Hematol 2010;38:233-45.

32. Deregibus MC, Cantaluppi V, Calogero R, et al. Endothelial progenitor cell derived microvesicles activate an angiogenic program in endothelial cells by a horizontal transfer of mRNA. Blood 2007;110:2440-8.

33. Deregibus MC, Tetta C, Camussi G. The dynamic stem cell microenvironment is orchestrated by microvesiclemediated transfer of genetic information. Histol Histopathol 2010;25:397-404.

34. Quesenberry PJ, Aliotta J, Deregibus MC, et al. Role of extracellular RNA-carrying vesicles in cell differentiation and reprogramming. Stem Cell Res Ther 2015;6:153.

35. Ratajczak J, Miekus K, Kucia M, et al. Embryonic stem cell-derived microvesicles reprogram hematopoietic progenitors: evidence for horizontal transfer of mRNA and protein delivery. Leukemia 2006;20:847-56.

36. Quesenberry PJ, Dooner MS, Aliotta JM. Stem cell plasticity revisited: the continuum marrow model and phenotypic changes mediated by microvesicles. Exp Hematol 2010;38:581-92.

37. Fatima F, Ekstrom K, Nazarenko I, et al. Non-coding RNAs in mesenchymal stem cell-derived extracellular vesicles: Deciphering regulatory roles in stem cell potency, inflammatory resolve and tissue regeneration. Front Genet 2017 8:161.

38. Baglio SR, Rooijers K, Koppers-Lalic D, et al. Human bone marrow- and adipose-mesenchymal stem cells secrete 
exosomes enriched in distinctive miRNA and tRNA species. Stem Cell Res Ther 2015;6:127.

39. De Luca L, Trino S, Laurenzana I, et al. MiRNAs and piRNAs from bone marrow mesenchymal stem cell extracellular vesicles induce cell survival and inhibit cell differentiation of cord blood hematopoietic stem cells: a new insight in transplantation. Oncotarget 2016;7:6676-92.

40. Billing AM, Ben Hamidane H, Dib SS, et al. Comprehensive transcriptomic and proteomic characterization of human mesenchymal stem cells reveals source specific cellular markers. Sci Rep 2016;6:21507.

41. Collino F, Deregibus MC, Bruno S, et al. Microvesicles derived from adult human bone marrow and tissue specific mesenchymal stem cells shuttle selected pattern of miRNAs. PLoS One 2010;5:e11803.

42. Guo L, Zhao RC, Wu Y. The role of microRNAs in selfrenewal and differentiation of mesenchymal stem cells. Exp Hematol 2011;39:608-16.

43. Pauli A, Rinn JL, Schier AF. Non-coding RNAs as regulators of embryogenesis. Nat Rev Genet 2011;12:136-49.

44. Fatica A, Bozzoni I. Long non-coding RNAs: new players in cell differentiation and development. Nat Rev Genet 2014;15:7-21.

45. Perry RB, Ulitsky I. The functions of long noncoding RNAs in development and stem cells. Development 2016;143:3882-94.

46. Pasquinelli AE, Ruvkun G. Control of developmental timing by micrornas and their targets. Annu Rev Cell Dev Biol 2002;18:495-513.

47. Carrington JC, Ambros V. Role of microRNAs in plant and animal development. Science 2003;301:336-8.

48. Ambros V. MicroRNA pathways in flies and worms: growth, death, fat, stress, and timing. Cell 2003;113:673-6.

49. Marson A, Levine SS, Cole MF, et al. Connecting microRNA genes to the core transcriptional regulatory circuitry of embryonic stem cells. Cell 2008;134:521-33.

50. Gangaraju VK, Lin H. MicroRNAs: key regulators of stem cells. Nat Rev Mol Cell Biol 2009;10:116-25.

51. Wei B, Wei W, Zhao B, et al. Long non-coding RNA HOTAIR inhibits miR-17-5p to regulate osteogenic differentiation and proliferation in nontraumatic osteonecrosis of femoral head. PLoS One 2017;12:e0169097.

52. Judson RL, Babiarz JE, Venere M, et al. Embryonic stem cell-specific microRNAs promote induced pluripotency. Nat Biotechnol 2009;27:459-61.

53. Zhang W, Dong R, Diao S, et al. Differential long noncoding $\mathrm{RNA} / \mathrm{mRNA}$ expression profiling and functional network analysis during osteogenic differentiation of human bone marrow mesenchymal stem cells. Stem Cell Res Ther 2017;8:30.

54. Deng J, Yang M, Jiang R, et al. Long Non-Coding RNA HOTAIR Regulates the Proliferation, Self-Renewal Capacity, Tumor Formation and Migration of the Cancer Stem-Like Cell (CSC) Subpopulation Enriched from Breast Cancer Cells. PLoS One 2017;12:e0170860.

55. Dinger ME, Amaral PP, Mercer TR, et al. Long noncoding RNAs in mouse embryonic stem cell pluripotency and differentiation. Genome Res 2008;18:1433-45.

56. Shenoy A, Blelloch RH. Regulation of microRNA function in somatic stem cell proliferation and differentiation. Nat Rev Mol Cell Biol 2014;15:565-76.

57. Xu C, Zhang Y, Wang Q, et al. Long non-coding RNA GAS5 controls human embryonic stem cell self-renewal by maintaining NODAL signalling. Nat Commun 2016;7:13287.

58. Loewer S, Cabili MN, Guttman M, et al. Large intergenic non-coding RNA-RoR modulates reprogramming of human induced pluripotent stem cells. Nat Genet 2010;42:1113-7.

59. Hou J, Long H, Zhou C, et al. Long noncoding RNA Braveheart promotes cardiogenic differentiation of mesenchymal stem cells in vitro. Stem Cell Res Ther 2017;8:4.

60. Karimnia N, Rafehi H, Tuano N, et al. Current perspectives in Set7 mediated stem cell differentiation. Non-Coding RNA 2016;2:14.

61. Ivey KN, Srivastava D. MicroRNAs as regulators of differentiation and cell fate decisions. Cell Stem Cell 2010;7:36-41.

62. Flynn RA, Chang HY. Long noncoding RNAs in cellfate programming and reprogramming. Cell Stem Cell 2014;14:752-61.

63. Shi XF, Wang H, Kong FX, et al. Exosomal miR-486 regulates hypoxia-induced erythroid differentiation of erythroleukemia cells through targeting Sirt1. Exp Cell Res 2017;351:74-81.

64. Katsman D, Stackpole EJ, Domin DR, et al. Embryonic stem cell-derived microvesicles induce gene expression changes in Muller cells of the retina. PLoS One 2012;7:e50417.

65. Salvucci O, Jiang K, Gasperini P, et al. MicroRNA126 contributes to granulocyte colony-stimulating factorinduced hematopoietic progenitor cell mobilization by reducing the expression of vascular cell adhesion molecule 
1. Haematologica 2012;97:818-26.

66. Wu J, Qu Z, Fei ZW, et al. Role of stem cell-derived exosomes in cancer. Oncol Lett 2017;13:2855-66.

67. Grange C, Tapparo M, Kholia S, et al. The Distinct Role of Extracellular Vesicles Derived from Normal and Cancer Stem Cells. Current Stem Cell Reports 2017;3:218-24.

68. Nawaz M, Fatima F, Nazarenko I, et al. Extracellular vesicles in ovarian cancer: applications to tumor biology, immunotherapy and biomarker discovery. Expert Rev Proteomics 2016;13:395-409.

69. Morello M, Minciacchi VR, de Candia P, et al. Large oncosomes mediate intercellular transfer of functional microRNA. Cell Cycle 2013;12:3526-36.

70. Zhang L, Zhang S, Yao J, et al. Microenvironmentinduced PTEN loss by exosomal microRNA primes brain metastasis outgrowth. Nature 2015;527:100-4.

71. Takahashi K, Yan IK, Haga H, et al. Modulation of hypoxia-signaling pathways by extracellular linc-RoR. J Cell Sci 2014;127:1585-94.

72. Yang M, Chen J, Su F, et al. Microvesicles secreted by macrophages shuttle invasion-potentiating microRNAs into breast cancer cells. Mol Cancer 2011;10:117.

73. Kosaka N, Ochiya T. Unraveling the Mystery of Cancer by Secretory microRNA: Horizontal microRNA Transfer between Living Cells. Front Genet 2012;2:97.

74. Fong MY, Zhou W, Liu L, et al. Breast-cancer-secreted miR-122 reprograms glucose metabolism in premetastatic niche to promote metastasis. Nat Cell Biol 2015;17:183-94.

75. Felicetti F, De Feo A, Coscia C, et al. Exosome-mediated transfer of miR-222 is sufficient to increase tumor malignancy in melanoma. J Transl Med 2016;14:56.

76. Kogure T, Lin WL, Yan IK, et al. Intercellular nanovesicle-mediated microRNA transfer: a mechanism of environmental modulation of hepatocellular cancer cell growth. Hepatology 2011;54:1237-48.

77. Liu Y, Luo F, Wang B, et al. STAT3-regulated exosomal miR-21 promotes angiogenesis and is involved in neoplastic processes of transformed human bronchial epithelial cells. Cancer Lett 2016;370:125-35.

78. Achreja A, Zhao H, Yang L, et al. Exo-MFA - A 13C metabolic flux analysis to dissect tumor microenvironmentsecreted exosome contributions towards cancer cell metabolism. Metab Eng 2017.

79. Qi J, Zhou Y, Jiao Z, et al. Exosomes Derived from Human Bone Marrow Mesenchymal Stem Cells Promote Tumor Growth Through Hedgehog Signaling Pathway. Cell Physiol Biochem 2017;42:2242-54.

80. Vallabhaneni KC, Penfornis P, Dhule S, et al. Extracellular vesicles from bone marrow mesenchymal stem/stromal cells transport tumor regulatory microRNA, proteins, and metabolites. Oncotarget 2015;6:4953-67.

81. Sun X, Ma X, Wang J, et al. Glioma stem cells-derived exosomes promote the angiogenic ability of endothelial cells through miR-21/NEGF signal. Oncotarget 2017;8:36137-48.

82. Wang M, Zhao C, Shi H, et al. Deregulated microRNAs in gastric cancer tissue-derived mesenchymal stem cells: novel biomarkers and a mechanism for gastric cancer. $\mathrm{Br} \mathrm{J}$ Cancer 2014;110:1199-210.

83. Sanchez CA, Andahur EI, Valenzuela R, et al. Exosomes from bulk and stem cells from human prostate cancer have a differential microRNA content that contributes cooperatively over local and pre-metastatic niche. Oncotarget 2016;7:3993-4008.

84. Horiguchi H, Kobune M, Kikuchi S, et al. Extracellular vesicle miR-7977 is involved in hematopoietic dysfunction of mesenchymal stromal cells via poly $(\mathrm{rC})$ binding protein 1 reduction in myeloid neoplasms. Haematologica 2016;101:437-47.

85. De Veirman K, Wang J, Xu S, et al. Induction of miR146a by multiple myeloma cells in mesenchymal stromal cells stimulates their pro-tumoral activity. Cancer Lett 2016;377:17-24.

86. El-Saghir J, Nassar F, Tawil N, et al. ATL-derived exosomes modulate mesenchymal stem cells: potential role in leukemia progression. Retrovirology 2016;13:73.

87. Kumar B, Garcia M, Weng L, et al. Acute myeloid leukemia transforms the bone marrow niche into a leukemia-permissive microenvironment through exosome secretion. Leukemia 2017. [Epub ahead of print].

88. Godlewski J, Ferrer-Luna R, Rooj AK, et al. MicroRNA Signatures and Molecular Subtypes of Glioblastoma: The Role of Extracellular Transfer. Stem Cell Reports 2017;8:1497-505.

89. Rooj AK, Ricklefs F, Mineo M, et al. MicroRNA-Mediated Dynamic Bidirectional Shift between the Subclasses of Glioblastoma Stem-like Cells. Cell Rep 2017;19:2026-32.

90. Garnier D, Meehan B, Kislinger T, et al. Divergent evolution of temozolomide resistance in glioblastoma stem cells is reflected in extracellular vesicles and coupled with radiosensitization. Neuro Oncol 2017. [Epub ahead of print].

91. Figueroa J, Phillips LM, Shahar T, et al. Exosomes from Glioma-Associated Mesenchymal Stem Cells Increase the Tumorigenicity of Glioma Stem-like Cells via Transfer of miR-1587. Cancer Res 2017. [Epub ahead of print]. 
92. Rodriguez M, Silva J, Herrera A, et al. Exosomes enriched in stemness/metastatic-related mRNAS promote oncogenic potential in breast cancer. Oncotarget 2015;6:40575-87.

93. Bliss SA, Sinha G, Sandiford OA, et al. Mesenchymal Stem Cell-Derived Exosomes Stimulate Cycling Quiescence and Early Breast Cancer Dormancy in Bone Marrow. Cancer Res 2016;76:5832-44.

94. Ren D, Lin B, Zhang X, et al. Maintenance of cancer stemness by miR-196b-5p contributes to chemoresistance of colorectal cancer cells via activating STAT3 signaling pathway. Oncotarget 2017;8:49807-23.

95. Ono M, Kosaka N, Tominaga N, et al. Exosomes from bone marrow mesenchymal stem cells contain a microRNA that promotes dormancy in metastatic breast cancer cells. Sci Signal 2014;7:ra63.

96. Santos JC, Ribeiro ML, Sarian LO, et al. Exosomesmediate microRNAs transfer in breast cancer chemoresistance regulation. Am J Cancer Res 2016;6:2129-39.

97. Au Yeung CL, Co NN, Tsuruga T, et al. Exosomal transfer of stroma-derived miR2 1 confers paclitaxel resistance in ovarian cancer cells through targeting APAF1. Nat Commun 2016;7:11150.

98. Lou G, Song X, Yang F, et al. Exosomes derived from miR-122-modified adipose tissue-derived MSCs increase chemosensitivity of hepatocellular carcinoma. J Hematol Oncol 2015;8:122.

99. Conigliaro A, Costa V, Lo Dico A, et al. CD90+ liver cancer cells modulate endothelial cell phenotype through the release of exosomes containing H19 lncRNA. Mol Cancer 2015;14:155.

100.Wang S, Li X, Zhu R, et al. Lung cancer exosomes initiate global long non-coding RNA changes in mesenchymal stem cells. Int J Oncol 2016;48:681-9.

doi: 10.21037/sci.2017.10.04

Cite this article as: Nawaz M. Extracellular vesicle-mediated transport of non-coding RNAs between stem cells and cancer cells: implications in tumor progression and therapeutic resistance. Stem Cell Investig 2017;4:83.
101. Takahara K, Ii M, Inamoto T, et al. microRNA-145 Mediates the Inhibitory Effect of Adipose Tissue-Derived Stromal Cells on Prostate Cancer. Stem Cells Dev 2016;25:1290-8.

102. Reza AM, Choi YJ, Yasuda H, et al. Human adipose mesenchymal stem cell-derived exosomal-miRNAs are critical factors for inducing anti-proliferation signalling to A2780 and SKOV-3 ovarian cancer cells. Sci Rep 2016;6:38498.

103. Fonsato V, Collino F, Herrera MB, et al. Human liver stem cell-derived microvesicles inhibit hepatoma growth in SCID mice by delivering antitumor microRNAs. Stem Cells 2012;30:1985-98.

104. Fareh M, Almairac F, Turchi L, et al. Cell-based therapy using miR-302-367 expressing cells represses glioblastoma growth. Cell Death Dis 2017;8:e2713.

105. Katakowski M, Buller B, Zheng X, et al. Exosomes from marrow stromal cells expressing miR-146b inhibit glioma growth. Cancer Lett 2013;335:201-4.

106. Pakravan K, Babashah S, Sadeghizadeh M, et al. MicroRNA-100 shuttled by mesenchymal stem cellderived exosomes suppresses in vitro angiogenesis through modulating the mTOR/HIF-1alpha/VEGF signaling axis in breast cancer cells. Cell Oncol (Dordr) 2017. [Epub ahead of print].

107.Alcayaga-Miranda F, Gonzalez PL, Lopez-Verrilli A, et al. Prostate tumor-induced angiogenesis is blocked by exosomes derived from menstrual stem cells through the inhibition of reactive oxygen species. Oncotarget 2016;7:44462-77.

108. Lee JK, Park SR, Jung BK, et al. Exosomes derived from mesenchymal stem cells suppress angiogenesis by downregulating VEGF expression in breast cancer cells. PLoS One 2013;8:e84256. 